\title{
Real-World Results of Ocrelizumab Treatment for Primary Progressive Multiple Sclerosis
}

\author{
K. Daniels $\mathbb{D}^{1,2}$ P. B. van der Nat, ${ }^{1,2}$ S. T. F. M. Frequin, ${ }^{3}$ P. J. van der Wees, ${ }^{2}$ D. H. Biesma, ${ }^{1,4}$ \\ E. L. J. Hoogervorst, ${ }^{3}$ and E. M. W. van de Garde ${ }^{5,6}$ \\ ${ }^{1}$ Department of Value-Based Healthcare, St. Antonius Hospital, Utrecht/Nieuwegein, Netherlands \\ ${ }^{2}$ Radboud University Medical Center, Radboud Institute for Health Sciences, \\ Scientific Center for Quality of Healthcare (IQ healthcare), Netherlands \\ ${ }^{3}$ Department of Neurology, St. Antonius Hospital, Utrecht/Nieuwegein, Netherlands \\ ${ }^{4}$ Department of Internal Medicine, University Medical Centre Utrecht, Utrecht, Netherlands \\ ${ }^{5}$ Department of Clinical Pharmacy, St. Antonius Hospital, Utrecht/Nieuwegein, Netherlands \\ ${ }^{6}$ Division of Pharmacoepidemiology and Clinical Pharmacology, Faculty of Science, Utrecht University, Utrecht, Netherlands
}

Correspondence should be addressed to K. Daniels; k.daniels@antoniusziekenhuis.nl

Received 28 January 2020; Revised 8 May 2020; Accepted 23 May 2020; Published 15 June 2020

Academic Editor: Pasquale Calabrese

Copyright (c) 2020 K. Daniels et al. This is an open access article distributed under the Creative Commons Attribution License, which permits unrestricted use, distribution, and reproduction in any medium, provided the original work is properly cited.

\begin{abstract}
Background. Recently, ocrelizumab (Ocrevus ${ }^{\circledR}$ ) was approved for the treatment of primary progressive multiple sclerosis (PPMS) based on data from the ORATORIO clinical trial. Real-world data about the clinical effectiveness of ocrelizumab has yet to be gathered. Objective. The aim of this study was to provide data about the clinical effectiveness of ocrelizumab for patients diagnosed with PPMS in a real-world setting. Methods. We conducted a retrospective cohort study of all patients with PPMS who started ocrelizumab treatment $(n=21)$ in St. Antonius Hospital (Utrecht/Nieuwegein, the Netherlands) between April 2018 and December 31, 2018. Primary outcome was pre- versus post-ocrelizumab disability worsening rate (from 96 weeks prior to first ocrelizumab administration up to 24 weeks post first ocrelizumab administration). Results. Disability worsening rate while on treatment significantly differed (lower) from disability worsening rate in pre-treatment period $(Z=-2.81, p \leq .01)$. Three out of 17 patients showed a clinically relevant improvement in disability status after treatment start. Conclusion. Ocrelizumab can stabilize disability progression in patients with PPMS. Some patients even showed a clinically relevant improvement in disability status. Further research should help to identify which patients benefit most from ocrelizumab.
\end{abstract}

\section{Introduction}

Traditionally, outcomes of drug treatments are mostly assessed in randomized controlled trials (RCTs). However, very often, the FDA/EMA-approved indication for the administration of a drug treatment in clinical practice is eventually broader than the inclusion criteria of the RCT. Also, even within the inclusion criteria of the original RCT, the real-world patient population could be more heterogeneous than the patient population in a RCT $[1,2]$. Because of variances in age, disease activity, or comorbidity [3-5], a drug can be more effective, less effective, or even not effective in real-world clinical practice.
In the field of multiple sclerosis (MS), there is a growing interest in the use of real-world data to evaluate outcomes of drug treatments [6-11]. Some disease-modifying therapies (DMTs) for MS have proven their efficacy in randomized clinical trial (RCT) but were not always equally effective for patients in real-world clinical practice $[6,7]$. Recently, the first FDA- and EMA-approved drug, ocrelizumab (Ocrevus $^{\circledR}$ ), for the treatment of primary progressive MS (PPMS) was introduced. The ORATORIO clinical trial demonstrated the efficacy of ocrelizumab over placebo in a selected group of patients diagnosed with PPMS [12-14]. Primary efficacy endpoint was 12 -week confirmed disability progression after treatment initiation, with percentages of $32.9 \%$ versus $39.3 \%$ 
for ocrelizumab and placebo, respectively [12]. To the best of our knowledge, no data is available yet about the effectiveness of ocrelizumab in PPMS in regular practice. The aim of this study was to provide data about the clinical effectiveness of ocrelizumab for patients diagnosed with PPMS in a realworld setting.

\section{Method}

2.1. Design and Setting. This study is a retrospective cohort study conducted at St. Antonius Hospital (Utrecht/Nieuwegein, the Netherlands), a large nonacademic hospital in the center of the Netherlands. At St. Antonius Hospital, ocrelizumab was available to patients with PPMS since Dutch market access in April 2018.

2.2. Patients. We studied all patients, diagnosed with PPMS according to the most recent McDonald criteria [15], who started ocrelizumab treatment in the period from Dutch market access (April 2018) until the end of 2018. As this study was a "real-world study," no inclusion or exclusion criteria were applied beforehand. Baseline characteristics that were collected contained age, gender, time since MS symptoms, time since PPMS diagnosis, previously used DMTs, B-cell-targeted therapies or other immunosuppressive medications, presence of oligoclonal bands in cerebrospinal fluid or elevated IgG index, history of RRMS, SPMS, or PRMS, contraindications to MRI, and side effects from oral or intravenous glucocorticoids. Baseline was set on the date of the first ocrelizumab administration. Baseline characteristics were also used to determine whether our study population could have been eligible or not eligible for the ORATORIO clinical trial [12].

2.3. Procedure. As part of their clinical treatment, patients diagnosed with PPMS received two $300 \mathrm{mg}$ ocrelizumab injections via an intravenous infusion at St. Antonius Hospital 14 days apart. Treatment was administered in a day-care setting overseen by a neurologist. Following regular procedures, patients were seen by a neurologist every twelve weeks, both pre-ocrelizumab administration and post-ocrelizumab administration.

2.4. Study Outcomes and Data Collection. The primary outcome of this study was pre- versus post-ocrelizumab disability worsening rate (from 96 weeks prior to first ocrelizumab administration up to 24 weeks post first ocrelizumab administration). Disability status was measured in Expanded Disability Status Scale (EDSS) scores [16] by neurologists certified in EDSS rating. The EDSS scale is scored in 0.5 unit increments and ranges from 0 to 10 points, with 0 indicating "totally healthy" and 10.0 indicating "death due to MS." An increase of minimal 0.5 points on the EDSS scale was defined as disability progression. For comparison purposes to the ORATORIO study, the 12-week confirmed disability progression was collected in the pre-ocrelizumab treatment period among all patients whose EDSS scores were available from 96 weeks prior to first ocrelizumab administration up to first ocrelizumab administration (baseline) $(n=17)$. Patients of whom clinical history was not fully available due to a transfer from another hospital were not included in this comparison.

Data, i.e., treatment outcomes, were either retrieved directly from patient files or approximated from documented neurological examinations. Data was collected by $\mathrm{KD}$ and independently verified by SF who is certified in EDSS rating. In case of discrepant findings, consensus was reached. REDCap CRF was used to store extracted data, guaranteeing anonymity and privacy of the patient cohort during and after this study. In case of missing EDSS values, e.g., due to patients missing a 12-week outpatient visit, the mean EDSS score of the previous and next outpatient visit was manually imputed. All missing values (9.7\%) occurred in pre-ocrelizumab treatment period; no values were missing in post-ocrelizumab treatment.

2.5. Statistical Analysis. IBM SPSS (version 24.0) was used to execute statistical analyses. Descriptive statistics were used for baseline characteristics. To assess whether the postocrelizumab disability worsening rate (EDSS at 24 weeks post treatment minus EDSS at treatment/number of weeks) differed from the pre-ocrelizumab disability worsening rate (EDSS at treatment minus EDSS at 96 weeks prior to treatment/number of weeks), a paired Wilcoxon Signed-Rank Test was executed. To assess whether the real-world preocrelizumab population was clinically similar to the ORATORIO placebo arm, in terms of 12-week confirmed disability progression over time, a time-to-event curve was generated. External reference data regarding disability progression over time was extracted from the ORATORIO clinical trial [12] to create this graph. Theoretically, the level of disease progression in the pre-ocrelizumab period should match the level of disease progression as seen in the placebo group from ORATORIO. No sample size calculations were made beforehand because of the explorative nature of the study.

\section{Results}

3.1. Study Population and Cohort Characteristics. From April 2018 through the end of 2018, a total of 21 patients diagnosed with PPMS started ocrelizumab treatment. The characteristics of the patients at baseline are provided in Table 1. In short, the mean age was 52 years and patients were diagnosed on average 5 years before. The mean EDSS score at time of ocrelizumab start was $5.3(\mathrm{SD}=1.1)$. Based on the exclusion and inclusion criteria from the ORATORIO clinical trial [12], a total of $n=11$ patients in our study cohort could have been eligible for the ORATORIO clinical trial whereas $n=10$ patients would not have been eligible for the ORATORIO clinical trial. The main reasons for noneligibility were higher age $(n=6)$, previously used B-cell-targeted therapies or other immunosuppressives $(n=1)$, history of RRMS, SPMS, or PRMS diagnosis $(n=1)$, and shorter history of disease $(n=2)$ (see supplemental Table 1). History of RRMS, SPMS, or PRMS diagnosis involved one patient who was previously misdiagnosed with RRMS and had previously received a DMT (Table 1). The diagnosis of this patient was altered into PPMS by the neurologist based on the patient's 
TABLE 1: Baseline characteristics of real-world patient population $(n=21)$, displayed separately for patients who met the eligibility criteria for the ORATORIO clinical trial and patients who did not meet the eligibility criteria.

\begin{tabular}{|c|c|c|c|c|}
\hline & $\begin{array}{l}\text { ORATORIO not eligible } \\
\qquad(N=10)\end{array}$ & $\begin{array}{l}\text { Real world } \\
\text { ORATORIO eligible } \\
\qquad(N=11)\end{array}$ & $\begin{array}{c}\text { Total } \\
(N=21)\end{array}$ & $\begin{array}{c}\text { ORATORIO }^{\mathrm{a}} \\
\text { Ocrelizumab }^{(N=488)} \\
\end{array}$ \\
\hline \multicolumn{5}{|l|}{ Age (yr) } \\
\hline Mean (SD) & $54.90(8.03)$ & $49.55(3.04)$ & $52.09(6.42)$ & $44.7(7.9)$ \\
\hline Median (range) & $56.50(37-67)$ & $49.00(46-54)$ & $53.00(37-67)$ & $46.0(20-56)$ \\
\hline Female sex-no. (\%) & $5(50.0)$ & $9(81.8)$ & $14(66.7)$ & $237(48.6)$ \\
\hline \multicolumn{5}{|l|}{ Time since onset of MS symptoms (yr) } \\
\hline Mean (SD) & $6.76(4.16)$ & $7.42(4.70)$ & $7.11(4.35)$ & $6.7(4.0)$ \\
\hline Median (range) & $6.04(.63-12.47)$ & $5.66(1.95-14.53)$ & $5.95(.63-14.53)$ & $6.0(1.1-32.9)$ \\
\hline \multicolumn{5}{|l|}{ Time since diagnosis of PPMS (yr) } \\
\hline Mean (SD) & $4.45(4.42)$ & $5.32(4.39)$ & $4.9(4.31)$ & $2.9(3.2)$ \\
\hline Median (range) & $3.30(.23-12.47)$ & $3.75(.21-14.53)$ & $3.75(.21-14.53)$ & $1.6(.1-16.8)$ \\
\hline $\begin{array}{l}\text { No previous use of disease-modifying } \\
\text { therapy-no. }(\%)\end{array}$ & $9(90.0)$ & $11(100)$ & $20(95.2)$ & $433(88.7)$ \\
\hline If yes: dimethyl fumarate & $1(10.0)$ & $0(0)$ & $1(4.8)$ & NA \\
\hline \multicolumn{5}{|l|}{ Score on EDSS } \\
\hline Mean (SD) & $4.9(.99)$ & $5.72(1.15)$ & $5.33(1.13)$ & $4.7(1.2)$ \\
\hline Median (range) & $4.75(3.5-6.5)$ & $6.50(3.5-6.5)$ & $6.0(3.5-6.5)$ & $4.5(2.5-7.0)$ \\
\hline
\end{tabular}

${ }^{\mathrm{a} O R A T O R I O}$ data were adapted from Montalban et al. [12].

clinical disease course more than one year prior to ocrelizumab administration. All patients fulfilled the FDA/ EMA-approved indication for ocrelizumab at time of administration, and in none of the cases, infusion-related reactions were recorded (compared to $39.9 \%$ infusionrelated reactions in ORATORIO trial [12]). Seventeen patients reached the 24-week follow-up point. For other patients, treatment was administered too recently to already report on 24-week EDSS scores $(n=2)$ or there was not enough historical data available due to the patient coming to the clinic for a second opinion or referral $(n=4)$ or both $(n=1)$.

Figure 1 displays patients' clinical disease course in two years (96 weeks) before treatment. During this pretreatment period, $76.5 \%$ of the real-world patient population showed 12-week confirmed disability progression compared to approximately $27 \%$ of the ORATORIO placebo group [12].

3.2. Effectiveness of Ocrelizumab. Primary outcome of this study was pre- versus post-ocrelizumab disability worsening rate (Figure 2). Disability worsening rate in pre-treatment period significantly differed from disability worsening rate while on treatment $(Z=-2.81, p \leq .01)$, indicating that the disability progression rate per 12 weeks was lower after the first ocrelizumab administration $(M=-.06, \mathrm{SD}=.29)$ than before treatment $(M=.09, \mathrm{SD}=.09)$. This suggests that ocrelizumab stabilized the disability progression. Furthermore, three out of 17 patients indicated that their disability status improved over time after treatment administration. One patient who gained health by 2.5 EDSS points had improved in walking ability (walker vs. ambulatory without aid), walking distance ( $\geq 20$ meters with aid vs. $\geq 500$ meters without aid), and balance. The other two patients who gained health by 0.5 EDSS points also reported an increase in walking ability (constant bilateral assistance vs. unilateral assistance) or walking distance ( $\geq 200$ meters vs. $\geq 300$ meters).

\section{Discussion}

This study provides first insight in the effect of ocrelizumab for the treatment of PPMS in a real-world clinical setting. This real-world data study confirmed that ocrelizumab can stabilize disability progression in patients with PPMS. Interestingly, three out of 17 patients even showed clinically relevant improvement in disability status. Ocrelizumab appears to be effective even though this real-world study population seems to have a more rapidly worsening form of PPMS than the study population in the ORATORIO clinical trial.

To the best of our knowledge, this study is the first to provide data about the real-world clinical effectiveness of ocrelizumab in patients with PPMS. This study used an unselected cohort, consisting of a heterogeneous group of patients that were all treated according to the FDA/EMA-classified indication for ocrelizumab. Additionally, it is interesting to see that only half of our real-world population could have been eligible for the ORATORIO clinical trial, meaning that the effect of ocrelizumab on this variety of patients has never been evaluated before. Further research is recommended to investigate whether the effect of ocrelizumab is different for ORATORIO eligible and not eligible patients. Unique in this study is the presentation of the absolute values of disability status (EDSS) over time, not just the cumulative probability of 12-week confirmed disability progression as was provided in the ORATORIO clinical trial. The absolute values of disability status provide additional insight in the severity of disability 


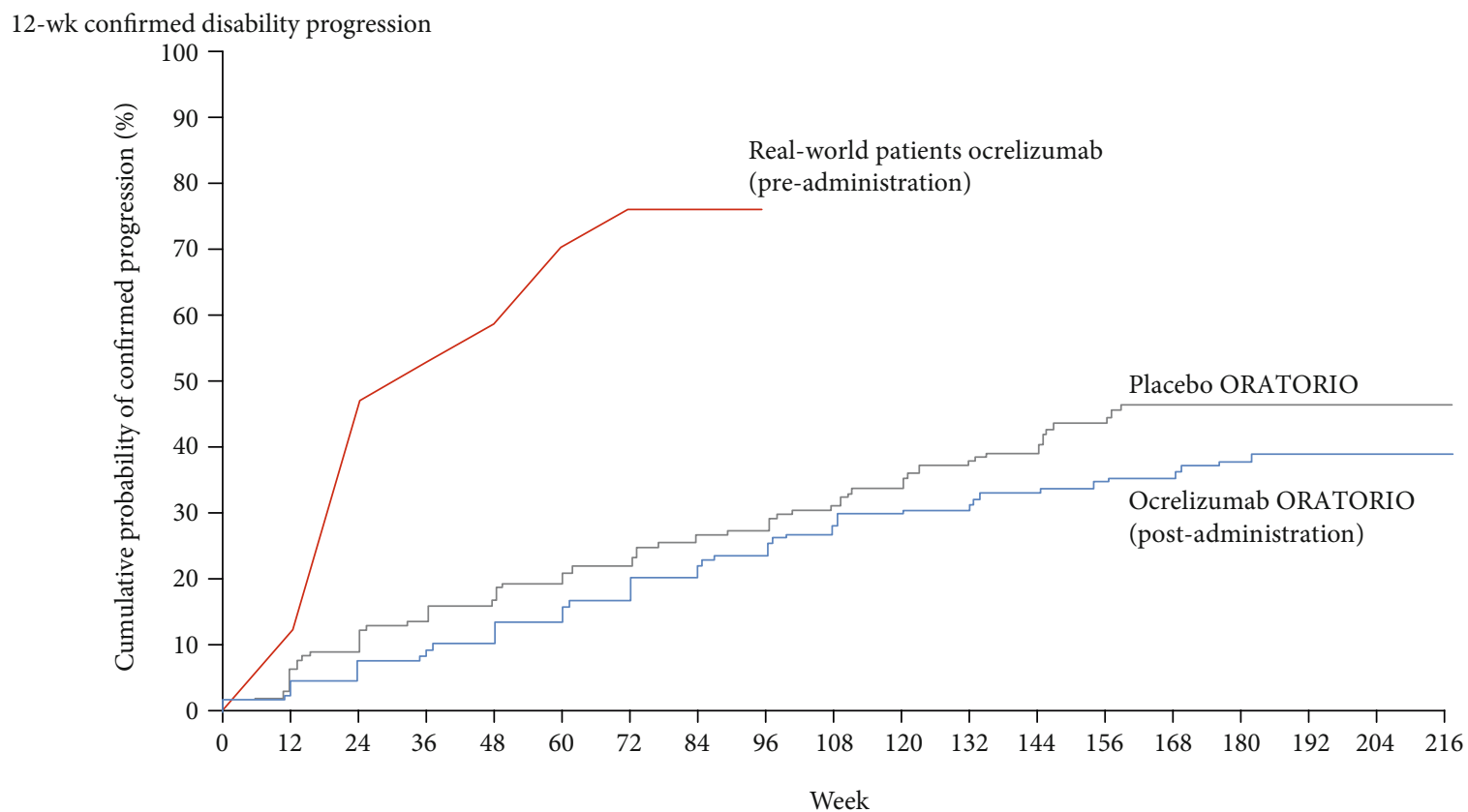

No. at risk; real-world patients

$\begin{array}{lllllllll}17 & 15 & 9 & 8 & 7 & 5 & 4 & 4 & 4\end{array}$

FIGURE 1: Time-to-event: 12-week confirmed disability progression of real-world pre-ocrelizumab population $(n=17)$ and the ORATORIO placebo arm. ORATORIO data were adapted from the original ORATORIO publication of Montalban et al. [12].

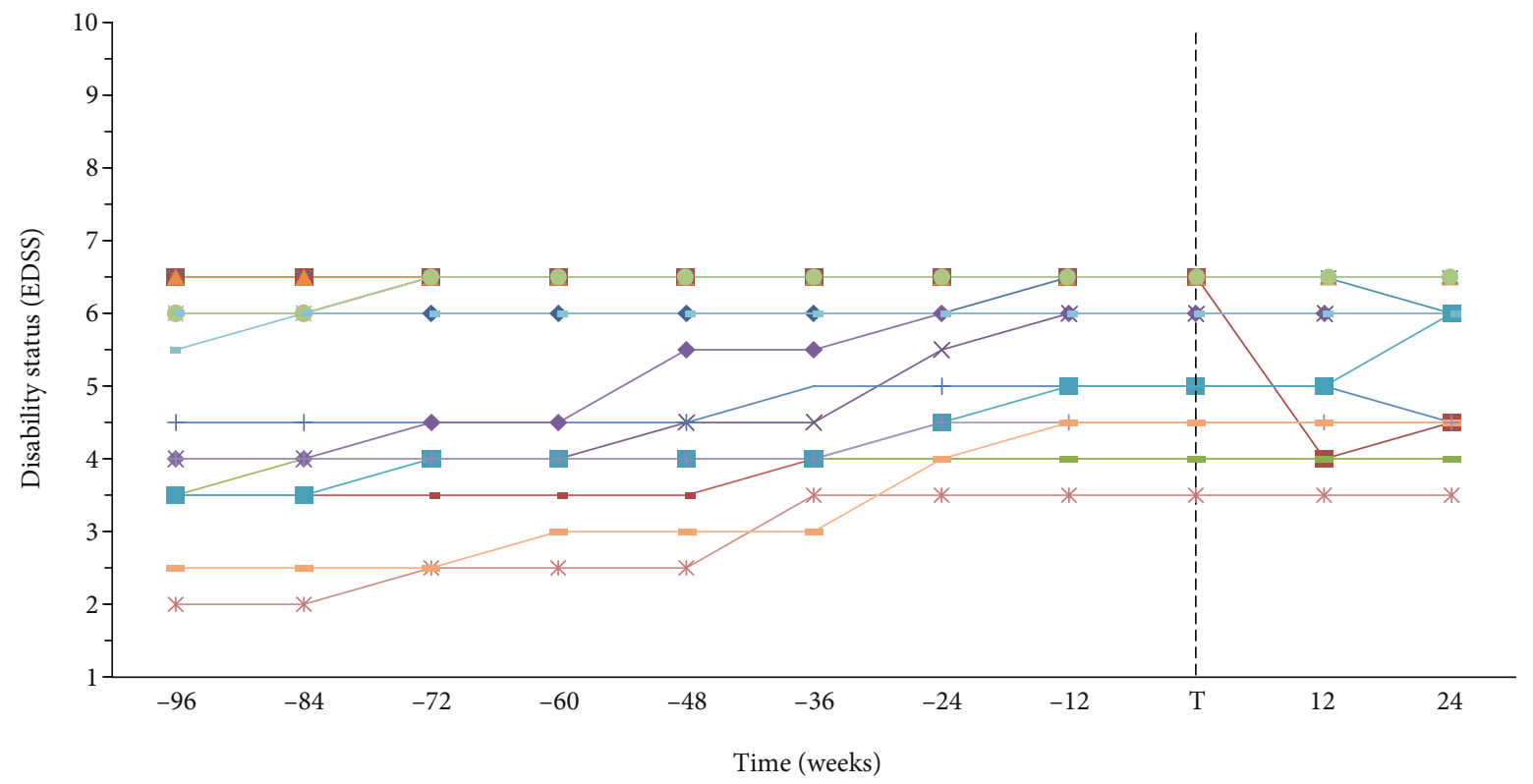

FIgURE 2: Pre- versus post-ocrelizumab disability worsening of patients diagnosed with PPMS $(N=17)$.

progression of every single patient. For example, a patient that starts with an EDSS score of 4.5 and reaches an EDSS score of 6.5 after two years has a different disability progression curve than a patient that starts with an EDSS score of 4.5 and reaches an EDSS score of 5.5 after two years. This insight is relevant to patients and can only be gained by presenting absolute values as was done in this study.
Although this study succeeded at reaching its aim, there were some limitations that should be considered when discussing this study's findings. First, one of the major limitations is the small sample size of this study due to the recent market introduction of ocrelizumab and the relatively low prevalence of PPMS. Therefore, our sample had insufficient power to perform subgroup analyses or to statistically 
compare our sample with that of the ORATORIO clinical trial. Second, due to the recent market introduction of ocrelizumab, the follow-up period of this study is relatively short (24 weeks). On the other hand, the ORATORIO clinical trial showed that the stabilizing effect of ocrelizumab on disability progression was primarily seen in the first 12 weeks [12]. After 12 weeks, the placebo group and the ocrelizumab group seemed to continue in approximately the same progression rate. This suggests that ocrelizumab does not slow progression in every patient but only in a subgroup. Possibly, these are our three out of 17 patients who experienced an improvement in disability status in the weeks following ocrelizumab administration. Unfortunately, the ORATORIO study report [12] does not provide data about how many patients' disability status improved and whether this happening was predictive for the 12-week confirmed disability progression outcome. Due to our short follow-up time, we could not explore this further at this moment. Other limitations of this study are standard for a retrospective study, being missing data, interpretation errors in medical records, and the absence of a control group, making this study a level IV evidence study [17].

In conclusion, this study showed that ocrelizumab can stabilize disability progression in patients with PPMS, even though this real-world study population seems to have a more rapidly worsening form of PPMS than the study population in the ORATORIO clinical trial. Our finding of some patients showing clinically relevant improvement in disability status is an important lead for further research into which patients benefit most from ocrelizumab.

\section{Data Availability}

Raw data were generated at St. Antonius Hospital. Derived data supporting the findings of this study are available from the corresponding author KD on request.

\section{Ethical Approval}

The protocol of this study was approved by the Medical research Ethics Committees United (MEC-U) (registration number: W18.218) and by the institutional review board of St. Antonius Hospital (registration number: R\&D/Z18.102). As this study only used historically routinely observed information from clinical practice, no informed consents were required. All data was documented anonymously and was safely stored.

\section{Conflicts of Interest}

The authors declared no potential conflicts of interest with respect to the research, authorship, and/or publication of this article.

\section{Authors' Contributions}

KD, PBVDN, and EMWVDG designed and initiated this study. KD collected data, analyzed data, and drafted the original manuscript. STFMF validated the outcome data.
PBVDN, EMWVDG, PJVDW, STFMF, DHB, and ELJH critically revised the original manuscript. All authors read and approved the final manuscript.

\section{Acknowledgments}

KD, PBVDN, STFMF, PJVDW and EMWVDG received funding for a VBHC investigator-institution initiated research for MS from Roche Nederland B.V. Funding paid to Institution. The present work was not supported by that funding.

\section{Supplementary Materials}

Supplemental Table 1: eligibility of real-world population in St. Antonius Hospital $(n=21)$ to ORATORIO clinical trial, separated for each eligibility criteria. (Supplementary Materials)

\section{References}

[1] R. E. Sherman, S. A. Anderson, G. J. Dal Pan et al., "Real-world evidence - what is it and what can it tell us?," The New England Journal of Medicine, vol. 375, no. 23, pp. 2293-2297, 2016.

[2] Food and Drug Administration, "Use of real-world evidence to support regulatory decision-making for medical devices: draft guidance for industry and Food and Drug Administration staff," 2017, January 2019, https://www.fda.gov/downloads/ MedicalDevices/DeviceRegulationandGuidance/ GuidanceDocuments/UCM513027.pdf.

[3] M. Trojano, M. Tintore, X. Montalban et al., "Treatment decisions in multiple sclerosis - insights from real-world observational studies," Nature Reviews Neurology, vol. 13, no. 2, pp. 105-118, 2017.

[4] P. M. Rothwell, "External validity of randomised controlled trials: "to whom do the results of this trial apply?"," The Lancet, vol. 365, no. 9453, pp. 82-93, 2005.

[5] E. A. Mills, J. A. Begay, C. Fisher, and Y. Mao-Draayer, "Impact of trial design and patient heterogeneity on the identification of clinically effective therapies for progressive MS," Multiple Sclerosis, vol. 24, no. 14, pp. 1795-1807, 2018.

[6] B. Moss, E. Utigard, L. Baldassari, J. Cohen, and D. Ontaneda, "Real-world experience with ocrelizumab," Neurology, vol. 90, article P6.356, Supplement 15, 2018.

[7] N. Vu, M. Bradshaw, H. Moses, S. Sriram, and S. Pawat, "Efficacy and tolerability of fingolimod, dimethylfumarate, and teriflunomide in patients with multiple sclerosis: real world experience from a single center," Neurology, vol. 86, article P6168, Supplement 16, 2016.

[8] S. H. Kim, M. S. Park, W. Kim et al., "Real-world effectiveness of disease-modifying therapies in Korean patients with relapsing multiple sclerosis," Journal of Clinical Neurology, vol. 15, no. 1, pp. 20-26, 2019.

[9] R. Zivadinov, J. Medin, N. Khan et al., "Impact of fingolimod on clinical and magnetic resonance imaging outcomes in routine clinical practice: a retrospective analysis of the multiple sclerosis, clinical and MRI outcomes in the USA (MS-MRIUS) study," Multiple Sclerosis and Related Disorders, vol. 27, pp. 65-73, 2019.

[10] T. Ziemssen, N. Ashtamker, S. Rubinchick, V. Knappertz, and G. Comi, "Long-term safety and tolerability of glatiramer 
acetate $20 \mathrm{mg} / \mathrm{ml}$ in the treatment of relapsing forms of multiple sclerosis," Expert Opinion on Drug Safety, vol. 16, no. 2, pp. 247-255, 2017.

[11] L. Prosperini, P. Annovazzi, L. Boffa et al., "No evidence of disease activity (NEDA-3) and disability improvement after alemtuzumab treatment for multiple sclerosis: a 36-month real-world study," Journal of Neurology, vol. 265, no. 12, pp. 2851-2860, 2018.

[12] X. Montalban, S. L. Hauser, L. Kappos et al., "Ocrelizumab versus placebo in primary progressive multiple sclerosis," The New England Journal of Medicine, vol. 376, no. 3, pp. 209220, 2017.

[13] L. Mayer, L. Kappos, M. K. Racke et al., “Ocrelizumab infusion experience in patients with relapsing and primary progressive multiple sclerosis: results from the phase 3 randomized OPERA I, OPERA II, and ORATORIO studies," Multiple Sclerosis and Related Disorders, vol. 30, pp. 236-243, 2019.

[14] E. J. Fox, C. Markowitz, A. Applebee et al., "Ocrelizumab reduces progression of upper extremity impairment in patients with primary progressive multiple sclerosis: findings from the phase III randomized ORATORIO trial," Multiple Sclerosis, vol. 24, no. 14, pp. 1862-1870, 2018.

[15] A. J. Thompson, B. L. Banwell, F. Barkhof et al., "Diagnosis of multiple sclerosis: 2017 revisions of the McDonald criteria," The Lancet Neurology, vol. 17, no. 2, pp. 162-173, 2018.

[16] J. F. Kurtzke, "Rating neurologic impairment in multiple sclerosis: an expanded disability status scale (EDSS)," Neurology, vol. 33, no. 11, pp. 1444-1452, 1983.

[17] G. H. Guyatt, D. L. Sackett, J. C. Sinclair, R. Hayward, D. J. Cook, and R. J. Cook, "Users' guides to the medical literature," Journal of the American Medical Association, vol. 274, no. 22, pp. 1800-1804, 1995. 